\title{
GENERATION MEAN ANALYSIS FOR DISEASE RESISTANCE, YIELD AND ITS COMPONENTS IN THREE CROSSES OF FABA BEAN (Vicia faba L.) \\ Abo Mostafa, R.A.I. ${ }^{1}$; E.A.D. Sarhan ${ }^{2}$ and Zeinab, E. Ghareeb ${ }^{3}$ \\ 1.Food Legumes Program, Field Crops Research Institute, ARC, Egypt. 2.Plant Pathology Research Institute, ARC, Egypt. \\ 3.Central Laboratory for design and Statistical Analysis Research, ARC, Egypt.
}

\begin{abstract}
The present study was conducted during 2011/12, 2012/13 and 2013/14 seasons at Sakha Agricultural Research Station, ARC, Kafr El-Sheikh Governorate, Egypt. Quantitative analyses were done by crossing between four faba bean (Vicia faba L.) parental genotypes namely Giza 40, Giza 429, Sakha 1 and Triple white (TW) to produce three crosses namely; Giza 40 x Giza 429 (I), Sakha 1 x Giza 429 (II) and Giza 429 x TW (III). The parental genotypes could be arranged into two groups. The first group included Sakha 1 where it is considered as the most resistant group to foliage diseases i.e, chocolate spot Botrytis fabae (Sard) and rust (Uromyces viciaefabae, Pers.) Schrot., and high yielding ability. The second group included Giza 40 , Giza 429 and TW as susceptible genotypes with low yielding potentiality. Generation mean analysis was used to estimate genetic variance components in the crosses for nine traits. Heterosis over mid and better parent for all traits were highly significant except plant height in the first (Giza 40 x Giza 429) and second (Sakha 1 x Giza 429 crosses relative to mid parent, in the first cross (Giza $40 \times$ Giza 429) relative to better parent, also the first cross had non-significant for no. of branches/plant and reaction to rust relative to mid parent and for no. of pods/plant relative to better parent. Also, the second (Sakha 1 x Giza 429) and third crosses (Giza 429 x TW) showed nonsignificance for chocolate spot relative to mid-parent and for 100 seed weight relative to better parent. The inbreeding depressing estimate was positive significant and/or highly significant for no. of branches/plant and no. of pods/plant, while it was negative significant for no. of seeds/pod in the three crosses. Heritability values in broad sense were generally higher than the corresponding values in narrow sense in all crosses for all traits. The additive genes seems to apply an improvement role of the inheritance of most of traits especially no. of pods/plant and no. of seeds/pod in the second cross; (Sakha 1 x Giza 429), where the differences between broad and narrow sense heritability were closes. The indirect selection in the progeny of this cross (Sakha $1 \mathrm{x}$ Giza 429) and direct selection in the progeny of other crosses would be fruitful due to the high values of narrow sense heritability and the prediction genetic advance in these crosses. The additive type (a) was significant positive and negative in all of crosses for most traits except no. of branches/plant and no. of seeds/pod in the first (Giza 40 x Giza 429) and third (Giza 429 x TW) crosses. For dominance effect (d) it was higher in magnitude than that of additive type of gene effects. The additive $x$ additive (aa) gene effect was highly significant in most crosses for all traits, except in a few cases. However, highly significant positive and negative epistatic gene action (ad) was observed in most crosses for most traits. The same trend was found with respect to dominance $x$ dominance $(\mathrm{dd})$ where positive and negative significant were observed for most crosses for most traits.
\end{abstract}




\section{INTRODUCTION}

Faba bean (Vicia faba L.) is one of the most important grain legumes in prone regions of North and East Africa, especially in Egypt. It play an important role in world agriculture, owing to its high protein content, ability to fix atmospheric nitrogen, capacity to grow and yield well on marginal lands (Al-Ghamdi, 2007).

One of the main reasons is the unreliable yields, mainly due to susceptibility of the crop to pests and diseases. A number of aerial fungi, together with soil-borne pathogens associated with foot and root rot complexes, nematodes, parasitic weeds and viruses may cause severe diseases in faba bean crop (Stoddard et al., 2010).

Faba bean (Vicia faba L.) crop has attracted the attention of most plant breeders to its yield because the importance of the crop for both human and animal nutrition. Foliage diseases chocolate spot Botrytis fabae (Sard) and rust Uromyces viciae-fabae (Pers.) Schrot., are the most destructive leaf disease of faba bean crop in the world. The losses as a result of foliage diseases were estimated to be more than $55 \%$ for susceptible cultivar Rebaya40 which was left for natural infection at Sakha (Mohamed et al., 1980).

The plant breeder is interested in estimating gene effects in order to formulate the most advantageous breeding procedures for improving the genetic material (Abdelmula, et al., 1999 and Bond et al., 1994)..

Six population analysis suggested by Gamble (1962) considered as the most important analysis method which supply the breeder by the information about the nature of gene action, heterosis, inbreeding depression, heritability and predicted genetic gain from selection for given characters.

The aim of the present investigation is to obtain useful information about gene action of foliage diseases, yield and its components as well as the extent of heterosis, inbreeding depression, heritability and genetic advance in the three faba bean crosses.

\section{MATERIALS AND METHODS}

\section{Field experiments:}

The present study was carried out during 2011/12, 2012/13 and 2013/14 seasons at Sakha Agricultural Research Station, ARC, Kafr ElSheikh Governorate, Egypt. Four faba bean genotypes (Vicia faba L.) were chosen for this study on the basis of genetic diversity and origin (Table 1). These genotypes were Giza40, Giza 429, Sakha1 and Triple white (TW). In 2011/12 growing season, these genotypes were sown and crossed under screen house to produce the F1 seeds of each three crosses: Cross I (Giza 40 x Giza 429), Cross II (Sakha 1 x Giza 429) and Cross III (Giza 429 x TW). In 2012/13 growing season, some of F1 plants for each cross and their parents were backcrossed to corresponding its two parents to obtain the backcrosses BC1 (F1 x P1) and BC2 (F1 x P2) generations. Some of the F1 plants were selfed to produce $F_{2}$ seeds and the others kept as $F 1, s$. 
In 2013/14 growing season, the six population seeds, i.e. P1, P2, F1, $F_{2}, B C 1$ and $B C 2$ of the three crosses were sown in a randomized complete block design with three replications, under natural infection of early sowing dates in November $1^{\text {st }}$ (early date). The plants were grown in ridges of two meters length and $60 \mathrm{~cm}$ width. Hills were spaced $20 \mathrm{~cm}$ apart with one seed per hill. Plots varied in size;25 rows for $F_{2}, 15$ rows for $B C 1$ and $B C 2$ and 3 rows for P1, P2 and F1. All cultural practices were done as usual with ordinary faba bean culture. Data were taken on plants of six populations in each cross for the following characters.

Table (1): The pedigree, diseases reaction and agronomic characters of four parental faba bean varieties used in the present study.

\begin{tabular}{|c|c|c|c|c|c|c|}
\hline \multirow{2}{*}{ Parent } & \multirow{2}{*}{ Genotype } & \multirow{2}{*}{ Pedigree } & \multirow{2}{*}{$\begin{array}{l}\text { Disease } \\
\text { reaction }\end{array}$} & \multirow{2}{*}{$\begin{array}{c}\text { Earliness } \\
\text { of } \\
\text { maturity }\end{array}$} & \multicolumn{2}{|c|}{$\begin{array}{l}\text { Agronomic } \\
\text { characters }\end{array}$} \\
\hline & & & & & \begin{tabular}{|c|}
$\begin{array}{c}\text { Seed coat } \\
\text { color }\end{array}$ \\
\end{tabular} & Seed size \\
\hline P1 & Giza40 & $\begin{array}{c}\text { Derived from } \\
\text { Rebaya } 40 \\
\end{array}$ & *H.S & Medium & Light brown & Medium \\
\hline P2 & Giza429 & $\begin{array}{l}\text { Derived from } \\
\text { Giza402 }\end{array}$ & H.S. & Medium & Light brown & Medium \\
\hline P3 & Sakha1 & $\begin{array}{l}\text { Giza 716 x } \\
620 / 283 / 85\end{array}$ & $\mathrm{R}$ & Very early & Light brown & Medium \\
\hline P4 & \begin{tabular}{|c|}
$\begin{array}{c}\text { Triple White } \\
\text { (TW) }\end{array}$ \\
\end{tabular} & $\begin{array}{l}\text { Introduced from } \\
\text { Sudan }\end{array}$ & H.S & Early & White & Small \\
\hline
\end{tabular}

${ }^{*} \mathrm{HR}=$ Highly resistant , R = resistant, MR = moderately resistant, $\mathrm{S}=$ susceptible, H.S. = Highly susceptible

Plant height, no. of branches/plant, no. of pods/plant, no. of seeds/plant, seed yield/plant (g), no. of seeds/pod, 100- seed weight, (chocolate spot and rust severity) under the natural infection were the characters registred. The resistance to foliage diseases were determined as in Table (2) with the adjustment of grading system from 0 to 9 for the increasing lesion percentage of leaf, flower and stem area covered by lesions, according to the scale of Bernier et al. (1993).

Table (2):Chocolate spot and rust disease scales by Bernier et al. (1993)

\begin{tabular}{|c|c|}
\hline \multicolumn{2}{|r|}{ Chocolate spot scale } \\
\hline 1 & No disease symptoms or very small specks (highly resistance) \\
\hline 3 & Few small disease lesions (resistant) \\
\hline 5 & Some coalesced lesions, with some defoliation (moderately resistant) \\
\hline 7 & $\begin{array}{l}\text { Large coalesced sporulating lesions, } 50 \% \text { defoliation and some dead plants } \\
\text { (susceptible) }\end{array}$ \\
\hline 9 & $\begin{array}{l}\text { Extensive, heavy sporulation, stem girdling, blackening and death of more than } 80 \% \\
\text { of plants (highly susceptible) }\end{array}$ \\
\hline \multicolumn{2}{|r|}{ Rust scale } \\
\hline 1 & No pustules or very small non-sporulating flecks (highly resistant) \\
\hline 3 & $\begin{array}{l}\text { Few scattered pustules covering less than } 1 \% \text { of the leaf area, and few or no } \\
\text { pustules on stem (resistant) }\end{array}$ \\
\hline 5 & $\begin{array}{l}\text { Pustules common on leaves covering } 1-4 \% \text { of leaf area, little defoliation and some } \\
\text { pustules on stem (moderately resistant). }\end{array}$ \\
\hline 7 & $\begin{array}{l}\text { Pustules very common on leaves covering } 4-8 \% \text { of leaf area, some defoliation and } \\
\text { many pustules on stem (susceptible). }\end{array}$ \\
\hline 9 & $\begin{array}{l}\text { Extensive pustules on leave, petioles and stem covering } 8-10 \% \text { of leaf area, many } \\
\text { dead leaves and several defoliation (highly susceptible). }\end{array}$ \\
\hline
\end{tabular}




\section{Disease Assessment:}

Reaction to foliar diseases (The disease severity of chocolate spot and rust diseases) was recorded on mid February and mid March for chocolate spot and rust diseases, respectively, according to the disease scales by Bernier et al. (1993) presented in table (1).

\section{Statistical analysis.}

To determine the presence or absence of non- allelic interaction, scaling test as outlined by Mather (1949) was used. The quantities A, B, C and $D$ and their variances have been calculated to test adequacy of the additive- dominance model in each case.

Where:

$$
\begin{aligned}
& \mathrm{A}=\overline{\mathrm{B}} \mathrm{c}_{1}-\overline{\mathrm{p}_{1}-\overline{\mathrm{F}_{1}}}- \\
& \mathrm{B}=\overline{2 \mathrm{~B} c 2-\mathrm{P} 2-\overline{\mathrm{F}} 2} \\
& \mathrm{C}=\overline{4} \mathrm{~F}_{2}-\overline{2} \overline{\mathrm{F}}_{1}-\mathrm{P}_{1}-\overline{\mathrm{P}}_{2} \\
& \mathrm{D}=\overline{2} \overline{\mathrm{F}}_{2}-\overline{\mathrm{B}} \mathrm{C}_{1}-\overline{\mathrm{BC}}_{2}
\end{aligned}
$$

And

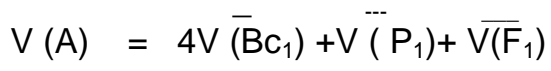

$\mathrm{A}(\mathrm{B})=4 \mathrm{~V}\left(\mathrm{Bc}_{2}\right)+\mathrm{V}\left(\mathrm{P}_{2}\right)+\overline{\mathrm{V}}\left(\mathrm{F}_{2}\right)$

$A(c)=1 \overline{6} \mathrm{~V}\left(F_{2}\right)+4 \bar{V}\left(F_{1}\right)+\bar{V}\left(P_{1}\right)+\bar{V}\left(P_{2}\right)$

$\mathrm{A}\{\mathrm{d}\}=\overline{4} \mathrm{~V}\left(\mathrm{~F}_{2}\right)+\mathrm{V}\left(\mathrm{BC}_{1}\right)+\mathrm{V}\left(\bar{B}_{2}\right)$

The standard error of $A, B, C$ and $D$ is worked out by taking square root, of respectively variances. The $\mathrm{t}$ - values are calculated by dividing the effects of $A, B, C$ and $D$ and $D$ by the respectively standard error. The calculated $t$-values were compared with tabulated value of $t$ at $5 \%$ levels of probability in each test, the degrees of freedom (df) is sum of (df) of various generation involved. The significance of $A$ and $B$ scales indicate the presence of all types of non - allelic gene interactions. The significance of $C$ scale suggests (dd) types of epistasis. The significance of $D$ scale reveals (aa) gene interactions, significance of $C$ and $D$ scale indicates (aa) and (dd) type of gene interactions (Singh and Narayanan, 1993).

Genetic analysis of generation means to give estimates of the types of gene effects were obtained using the relationships given by Gamble (1962).

Jinks and Jones (1958) however, used following formulae to estimate $\mathrm{m}$, a and $\mathrm{d}$ components in the absence of non- allelic interactions:

$m=\overline{1} / 2 p_{1}+\overline{1} / 2 p_{2}+\overline{4} F 1-2 \bar{B} C_{1}-2 \bar{B} C_{2}$ 
$d==\overline{1 / 2} p_{1}-\overline{1 / 2} p_{2}$

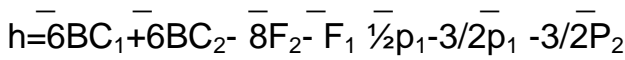

where, Their variances have been computed using following formulae:

$\mathrm{V}_{\mathrm{m}}=1 / 4 \overline{\mathrm{V}} \mathrm{P}_{1+1 / 4} \overline{\mathrm{V}} \mathrm{P}_{2+}+6 \overline{\mathrm{V}} \mathrm{F}_{1}+4 \overline{\mathrm{V}} \mathrm{BC}_{1}+\overline{4} \overline{\mathrm{VBC}} \mathrm{C}_{2}$

$\mathrm{V}_{\mathrm{d}}=1 / 4 \overline{\mathrm{V}} \mathrm{P}_{1+1 / 4} \overline{\mathrm{V}} \mathrm{P}_{2}$

And

$\mathrm{V}_{\mathrm{h}}=36 \mathrm{VBC}_{1}+36 \mathrm{VBC} \bar{C}_{2}+64 \mathrm{VF}_{2}+\mathrm{VF}_{1+} \overline{9} / 4 \mathrm{Vp}_{1}+9 / 4 \mathrm{Vp}_{2}$

- $\quad \mathrm{SE}(\mathrm{m})=(\mathrm{Vm})^{1 / 2}, \mathrm{SE}\{\mathrm{d}\}=(\mathrm{Vd})^{1 / 2}$, and $\mathrm{SE}\{\mathrm{h}\}=(\mathrm{Vh})^{1 / 2}$

- $\quad t(m)=m / S E(m), t\{d\}=d / S E\{d\}$ and $t\{h\}=h / S E\{h\}$

Broad + sense heritability $\left(\mathrm{H}^{2}\right)$ for $\mathrm{F}_{2}-$ generation was estimated based on the equation:

$$
H_{2}=\frac{V g}{V g+V e} \times 100
$$

The genetic variance $(\mathrm{Vg})$ and environmental $(\mathrm{Ve})$ were estimated according to Mansur et al.(1993) as follows:

$$
V_{e}=n_{e}^{-1}\left(n_{p 1} v_{p 1}+n_{p 2} v_{p 2}+n_{f 1} v_{f 1}\right)
$$

Where, ne $=n_{p 1}+n_{p 2}+n_{f 1}$ and $n_{p 1}, n_{p 2}$ and $n_{f 1}$ are the number of plants of $P_{1}, P_{2}$ and $F_{1}$ generations in each cross, respectively .

Narrow-sense heritability $\left(h^{2}\right)$ for $F_{2}{ }^{-}$generation was estimated as proposed by Warner (1952).

Where:

$\mathrm{H}^{2}=2 \mathrm{VF}_{2-}\left(\mathrm{VBC}_{1}+\mathrm{VBC}_{2}\right) / \mathrm{VF} 2 \times 100$

The phenotypic (PCV\%) and Genotypic (GCV\%) coefficient of variation were estimated as formulae developed by Burton (1952).

The expected genetic advance form selection $\left(G_{g}\right)$ was calculated as the formulae proposed by Johnson et al. (1955), using the selection differential $(K)$ equal 2.06 for $5 \%$ selection intensity and heritability in narrow sense.

The predicted genetic advance where the expected genetic gain upon selection was expressed as percentage of $F_{2}$ mean $(\mathrm{Ga} \%)$ was calculated following Miller et al.(1958).

The amount of heterosis was expressed as the percentage deviation of F1 mean performance from mid- parent and better parent. Inbreeding depression was calculated as the difference between the $F_{1}$ and $F_{2}$ means as 
a percentage of $F_{1}$. The "t " test was used to determine the significance of these deviations where the standard error (SE) was calculated as follows :

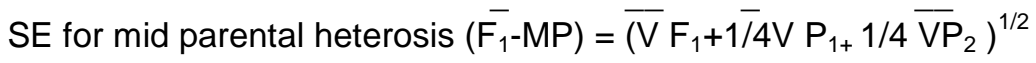

$\mathrm{SE}$ for better parental heterosis $\left(\overline{\mathrm{F}}_{1}-\mathrm{MP}\right)=\left(\overline{\mathrm{V}} \mathrm{F}_{1}+1 / 4 \overline{\mathrm{V}} \mathrm{P}_{1+}+1 / 4 \overline{\mathrm{V}} \mathrm{P}_{2}\right)^{1 /}$

$\mathrm{SE}$ for inbreeding depression $(\bar{F} \overline{1}-\mathrm{F} 2) \overline{-}\left(\mathrm{V} \overline{\mathrm{F}}_{1}+\mathrm{VF}_{2}\right)^{1 / 2}$

Where, the $t$ is the deviation /SE at the corresponding degrees of freedom.

\section{RESULTS AND DISCUSSION}

Data in Table (3) showed no. of plants, mean performance and variance of mean for the six populations of the three crosses for different traits. Significantly differences were observed among most genotypes for measured traits. The parental genotypes could be arranged into two groups; the first group included the genotype of Sakha1 which was considered to be the most resistant group to chocolate spot and rust with high yielding ability where the yield value per plant was $101.10 \mathrm{gm}$, in addition, it has less rating scale values of chocolate spot and rust reaction (3.01 and 3.25), respectively. The second group included Giza40, Giza 429 and Triple white which performed as susceptible genotypes it had high rating scale values of chocolate spot reaction $(6.16,6.23$ and 6.15$)$, also had high rating scale values of rust reaction $(6.61,6.56$ and 6.97), respectively, accompanied with low yielding ability $(91.53,80.86$ and $21.64 \mathrm{~g})$, respectively. The difference between the studied crosses with respect to foliar diseases resistance and yield component traits could be observed in Table (3), also significant genetic variance were detected for all traits in the three crosses and therefore genetic parameters were detected as reported by Khalil et al. (1993b) and Attia et al. (2006).

Genetic variances were detected for all traits in the three crosses and therefore, genetic parameters were detected as reported by Khalil et al. (1993) and Attia et al. (2006).. 
Table (3): Number of plants ( $n$ ), mean performance ( $x$ ) and variance of mean $\left(S^{2}\right)$ for studied traits in the three faba bean crosses.

\begin{tabular}{|c|c|c|c|c|c|c|c|c|}
\hline Traits & Crosses & $\begin{array}{l}\text { Statistical } \\
\text { parameter }\end{array}$ & $\mathbf{P}_{1}$ & $\mathbf{P}_{2}$ & $F_{1}$ & $F_{2}$ & $\mathrm{BC}_{1}$ & $\mathrm{BC}_{2}$ \\
\hline \multirow{3}{*}{$\begin{array}{l}\text { Plant } \\
\text { height }\end{array}$} & $\begin{array}{c}\text { Cross } 1 \\
\text { (Giza40 } \\
\text { xGiza429) }\end{array}$ & $\frac{\frac{n}{X}}{S^{2} \frac{X}{X}}$ & $\begin{array}{c}30 \\
130 \\
0.632 \\
\end{array}$ & $\begin{array}{c}30 \\
123.33 \\
0.720\end{array}$ & $\begin{array}{c}30 \\
128.17 \\
0.772\end{array}$ & $\begin{array}{c}250 \\
126.2 \\
0.381 \\
\end{array}$ & $\begin{array}{c}150 \\
129.12 \\
0.407\end{array}$ & $\begin{array}{c}150 \\
125.22 \\
0.498\end{array}$ \\
\hline & $\begin{array}{c}\text { Cross } 2 \\
\text { (Sakha1 x } \\
\text { Giza429) }\end{array}$ & $\frac{n}{S^{2} \frac{n}{X}}$ & $\begin{array}{c}30 \\
136.89 \\
0.979\end{array}$ & $\begin{array}{c}30 \\
123.33 \\
0.720\end{array}$ & $\begin{array}{c}30 \\
129.00 \\
1.038\end{array}$ & $\begin{array}{c}250 \\
126.00 \\
0.585\end{array}$ & $\begin{array}{c}150 \\
139.33 \\
0.643\end{array}$ & $\begin{array}{c}150 \\
132.11 \\
0.738\end{array}$ \\
\hline & $\begin{array}{c}\text { Cross } 3 \\
\text { (Giza } 429 \times \\
\text { TW) }\end{array}$ & $\frac{n}{S^{2} \frac{n}{X}}$ & $\begin{array}{c}30 \\
123.33 \\
0.720 \\
\end{array}$ & $\begin{array}{c}30 \\
103.50 \\
0.843 \\
\end{array}$ & $\begin{array}{c}30 \\
119.46 \\
0.717 \\
\end{array}$ & $\begin{array}{c}250 \\
120.12 \\
0.501 \\
\end{array}$ & $\begin{array}{c}150 \\
112.35 \\
0.574 \\
\end{array}$ & $\begin{array}{c}150 \\
115.68 \\
0.601 \\
\end{array}$ \\
\hline \multirow{3}{*}{$\begin{array}{l}\text { No. of } \\
\text { branches } \\
\text { plant }\end{array}$} & $\begin{array}{c}\text { Cross } 1 \\
\text { (Giza40 } \\
x \text { Giza429) }\end{array}$ & $\frac{n}{s^{2} \frac{n}{X}}$ & $\begin{array}{c}30 \\
3.57 \\
0.010\end{array}$ & $\begin{array}{c}30 \\
3.13 \\
0.015\end{array}$ & $\begin{array}{c}30 \\
3.43 \\
0.029 \\
\end{array}$ & $\begin{array}{c}250 \\
2.56 \\
0.008 \\
\end{array}$ & $\begin{array}{c}150 \\
3.41 \\
0.009 \\
\end{array}$ & $\begin{array}{c}150 \\
3.08 \\
0.011 \\
\end{array}$ \\
\hline & $\begin{array}{c}\text { Cross } 2 \\
\text { (Sakha1 x } \\
\text { Giza429) }\end{array}$ & $\frac{n}{s^{2} \bar{X}}$ & $\begin{array}{c}30 \\
3.50 \\
0.010 \\
\end{array}$ & $\begin{array}{c}30 \\
3.13 \\
0.015 \\
\end{array}$ & $\begin{array}{c}30 \\
4.07 \\
0.017 \\
\end{array}$ & $\begin{array}{c}250 \\
3.38 \\
0.010\end{array}$ & $\begin{array}{c}150 \\
4.62 \\
0.013 \\
\end{array}$ & $\begin{array}{c}150 \\
4.05 \\
0.012 \\
\end{array}$ \\
\hline & $\begin{array}{c}\text { Cross } 3 \\
\text { (Giza } 429 \times \\
\text { TW) }\end{array}$ & $\frac{n}{S^{2} \frac{n}{X}}$ & $\begin{array}{c}30 \\
3.13 \\
0.015 \\
\end{array}$ & $\begin{array}{c}30 \\
1.50 \\
0.013 \\
\end{array}$ & $\begin{array}{c}30 \\
3.42 \\
0.009 \\
\end{array}$ & $\begin{array}{c}250 \\
3.27 \\
0.017 \\
\end{array}$ & $\begin{array}{c}150 \\
2.72 \\
0.015 \\
\end{array}$ & $\begin{array}{c}150 \\
2.98 \\
0.020 \\
\end{array}$ \\
\hline \multirow{3}{*}{$\begin{array}{l}\text { No. of } \\
\text { pods/ plant }\end{array}$} & $\begin{array}{c}\text { Cross } 1 \\
\text { (Giza40 } \\
\times \text { Giza429) }\end{array}$ & $\frac{n}{s^{2} \frac{n}{X}}$ & $\begin{array}{c}30 \\
50.33 \\
0.267 \\
\end{array}$ & $\begin{array}{c}30 \\
37.7 \\
0.306 \\
\end{array}$ & $\begin{array}{c}30 \\
52.4 \\
0.376 \\
\end{array}$ & $\begin{array}{c}250 \\
38.9 \\
0.249 \\
\end{array}$ & $\begin{array}{c}150 \\
49.24 \\
0.301 \\
\end{array}$ & $\begin{array}{c}150 \\
39.47 \\
0.281 \\
\end{array}$ \\
\hline & $\begin{array}{c}\text { Cross } 2 \\
\text { (Sakha1 x } \\
\text { Giza429) }\end{array}$ & $\frac{\mathrm{n}}{\mathrm{s}^{2} \frac{\bar{X}}{\mathrm{X}}}$ & $\begin{array}{c}30 \\
39.97 \\
0.852 \\
\end{array}$ & $\begin{array}{c}30 \\
37.70 \\
0.306 \\
\end{array}$ & $\begin{array}{c}30 \\
45.73 \\
1.105\end{array}$ & $\begin{array}{c}250 \\
40.10 \\
1.264\end{array}$ & $\begin{array}{c}150 \\
39.87 \\
1.190 \\
\end{array}$ & $\begin{array}{c}150 \\
46.40 \\
1.261 \\
\end{array}$ \\
\hline & $\begin{array}{c}\text { Cross } 3 \\
\text { (Giza } 429 x \\
\text { TW) }\end{array}$ & $\frac{n}{s^{2} \frac{n}{X}}$ & $\begin{array}{c}30 \\
37.7 \\
0.306 \\
\end{array}$ & $\begin{array}{c}30 \\
23.48 \\
0.426 \\
\end{array}$ & $\begin{array}{c}30 \\
43.35 \\
0.704 \\
\end{array}$ & $\begin{array}{c}250 \\
41.25 \\
1.283 \\
\end{array}$ & $\begin{array}{c}150 \\
34.36 \\
1.282 \\
\end{array}$ & $\begin{array}{c}150 \\
33.7 \\
1.475 \\
\end{array}$ \\
\hline \multirow{3}{*}{$\begin{array}{l}\text { No. of } \\
\text { seeds/ } \\
\text { plant }\end{array}$} & $\begin{array}{c}\text { Cross } 1 \\
\text { (Giza40 } \\
\text { xGiza429) } \\
\end{array}$ & $\frac{\mathrm{n}}{\mathrm{X}}$ & $\begin{array}{c}30 \\
141.7 \\
0.587 \\
\end{array}$ & $\begin{array}{c}30 \\
118.01 \\
0.524\end{array}$ & $\begin{array}{c}30 \\
135.6 \\
0.555 \\
\end{array}$ & $\begin{array}{c}250 \\
120.12 \\
0.301 \\
\end{array}$ & $\begin{array}{c}150 \\
139.13 \\
0.341 \\
\end{array}$ & $\begin{array}{c}150 \\
121.11 \\
0.375 \\
\end{array}$ \\
\hline & $\begin{array}{l}\text { ss } 2 \\
\text { (Sakha1 } \\
\text { Giza429) } \\
\end{array}$ & $\frac{\text { Cron }}{\mathrm{s}^{2} \overline{\mathrm{X}}}$ & $\begin{array}{c}30 \\
126.00 \\
0.600\end{array}$ & $\begin{array}{c}30 \\
118.01 \\
0.524\end{array}$ & $\begin{array}{c}30 \\
140.93 \\
0.880\end{array}$ & $\begin{array}{c}250 \\
128.00 \\
1.017 \\
\end{array}$ & $\begin{array}{c}150 \\
141.44 \\
1.401 \\
\end{array}$ & $\begin{array}{c}150 \\
138.22 \\
1.321\end{array}$ \\
\hline & $\begin{array}{l}\text { Cross } 3 \\
(\text { Giza } 429 \quad x \\
\text { TW) }\end{array}$ & $\frac{\mathrm{n}}{\mathrm{S}^{2} \frac{\bar{X}}{\mathrm{X}}}$ & $\begin{array}{c}30 \\
118.01 \\
0.524\end{array}$ & $\begin{array}{c}30 \\
43.56 \\
0.621\end{array}$ & $\begin{array}{c}30 \\
126.12 \\
0.938\end{array}$ & $\begin{array}{c}250 \\
131.15 \\
0.521\end{array}$ & $\begin{array}{c}150 \\
75.18 \\
0.654\end{array}$ & $\begin{array}{c}150 \\
86.22 \\
0.541 \\
\end{array}$ \\
\hline \multirow{3}{*}{$\begin{array}{c}\text { Seed yield/ } \\
\text { plant }\end{array}$} & $\begin{array}{c}\text { Cross } 1 \\
\text { (Giza40 } \\
\times \text { Giza429) } \\
\end{array}$ & $\frac{\mathrm{n}}{\mathrm{s}^{2} \overline{\mathrm{X}}}$ & $\begin{array}{c}30 \\
91.53 \\
0.306 \\
\end{array}$ & $\begin{array}{c}30 \\
80.86 \\
0.299 \\
\end{array}$ & $\begin{array}{c}30 \\
94.66 \\
0.471 \\
\end{array}$ & $\begin{array}{c}250 \\
80.2 \\
0.441 \\
\end{array}$ & $\begin{array}{c}150 \\
85.17 \\
0.428 \\
\end{array}$ & $\begin{array}{c}150 \\
82.89 \\
0.521 \\
\end{array}$ \\
\hline & $\begin{array}{c}\text { Cross } 2 \\
\text { (Sakha1 x } \\
\text { Giza429) }\end{array}$ & $\frac{\mathrm{n}}{\mathrm{S}^{2} \overline{\mathrm{X}}}$ & $\begin{array}{c}30 \\
101.10 \\
0.591 \\
\end{array}$ & $\begin{array}{c}30 \\
80.86 \\
0.299 \\
\end{array}$ & $\begin{array}{c}30 \\
113.84 \\
0.539 \\
\end{array}$ & $\begin{array}{c}250 \\
98.40 \\
0.799 \\
\end{array}$ & $\begin{array}{c}150 \\
123.70 \\
0.889 \\
\end{array}$ & $\begin{array}{c}150 \\
103.22 \\
0.814 \\
\end{array}$ \\
\hline & $\begin{array}{c}\text { Cross } 3 \\
\text { (Giza } 429 \times \\
\text { TW) }\end{array}$ & $\frac{n}{S^{2} \frac{n}{X}}$ & $\begin{array}{c}30 \\
80.86 \\
0.333\end{array}$ & $\begin{array}{c}30 \\
21.64 \\
0.659 \\
\end{array}$ & $\begin{array}{c}30 \\
82.28 \\
0.706\end{array}$ & $\begin{array}{c}250 \\
86.30 \\
0.525 \\
\end{array}$ & $\begin{array}{c}150 \\
49.16 \\
0.594\end{array}$ & $\begin{array}{c}150 \\
52.41 \\
0.641 \\
\end{array}$ \\
\hline
\end{tabular}


Abo Mostafa, R.A.l. et al.

table 3 cont:

\begin{tabular}{|c|c|c|c|c|c|c|c|c|}
\hline Traits & Crosses & $\begin{array}{l}\text { Statistical } \\
\text { parameter }\end{array}$ & $\mathbf{P}_{1}$ & $\mathbf{P}_{2}$ & $F_{1}$ & $F_{2}$ & $\mathrm{BC}_{1}$ & $\mathrm{BC}_{2}$ \\
\hline \multirow{9}{*}{$\begin{array}{c}\text { No. of } \\
\text { seeds/ pod }\end{array}$} & \multirow{3}{*}{$\begin{array}{c}\text { Cross } 1 \\
\text { (Giza40 } \\
\times \text { Giza429) } \\
\end{array}$} & $\mathrm{n}$ & 30 & 30 & 30 & 250 & 150 & 150 \\
\hline & & $\overline{\mathrm{X}}$ & 2.82 & 3.21 & 2.41 & 3.09 & 2.83 & 3.07 \\
\hline & & $s^{2} \bar{X}$ & 0.004 & 0.003 & 0.004 & 0.005 & 0.005 & 0.004 \\
\hline & \multirow{3}{*}{$\begin{array}{c}\text { Cross } 2 \\
\text { (Sakha1 x } \\
\text { Giza429) } \\
\end{array}$} & $\mathrm{n}$ & 30 & 30 & 30 & 250 & 150 & 150 \\
\hline & & $\mathrm{X}$ & 3.15 & 3.21 & 3.08 & 3.19 & 3.55 & 2.98 \\
\hline & & $s^{2} \bar{X}$ & 0.004 & 0.003 & 0.005 & 0.003 & 0.002 & 0.002 \\
\hline & \multirow{3}{*}{$\begin{array}{c}\text { Cross } 3 \\
\text { (Giza } 429 \text { x } \\
\text { TW) }\end{array}$} & $\mathrm{n}$ & 30 & 30 & 30 & 250 & 150 & 150 \\
\hline & & $\underline{X}$ & 3.21 & 1.86 & 3.05 & 3.18 & 2.19 & 2.56 \\
\hline & & $s^{2} \bar{X}$ & 0.003 & 0.009 & 0.013 & 0.012 & 0.012 & 0.014 \\
\hline \multirow{9}{*}{$\begin{array}{c}100 \text { seed } \\
\text { weight }\end{array}$} & \multirow{3}{*}{$\begin{array}{c}\text { Cross } 1 \\
\text { (Giza40 } \\
\text { xGiza429) }\end{array}$} & $\mathrm{n}$ & 30 & 30 & 30 & 250 & 150 & 150 \\
\hline & & $\mathrm{X}$ & 64.59 & 68.51 & 69.81 & 66.77 & 68.40 & 68.44 \\
\hline & & $s^{2} \bar{X}$ & 0.364 & 0.385 & 0.419 & 0.273 & 0.270 & 0.348 \\
\hline & \multirow{3}{*}{$\begin{array}{c}\text { Cross } 2 \\
(\text { Sakha1 x } \\
\text { Giza429) } \\
\end{array}$} & $\mathrm{n}$ & 30 & 30 & 30 & 250 & 150 & 150 \\
\hline & & $\mathrm{X}$ & 80.24 & 68.51 & 80.78 & 76.88 & 87.46 & 74.61 \\
\hline & & $s^{2} \bar{X}$ & 0.753 & 0.385 & 0.606 & 1.012 & 0.848 & 1.260 \\
\hline & \multirow{3}{*}{$\begin{array}{c}\text { Cross } 3 \\
\text { (Giza } 429 x \\
\text { TW) }\end{array}$} & $\mathrm{n}$ & 30 & 30 & 30 & 250 & 150 & 150 \\
\hline & & X & 68.51 & 49.68 & 65.24 & 65.8 & 65.39 & 60.79 \\
\hline & & $s^{2} \overline{\mathbf{X}}$ & 0.385 & 0.608 & 1.435 & 0.405 & 0.428 & 0.476 \\
\hline \multirow{9}{*}{$\begin{array}{c}\text { Chocolate } \\
\text { spot }\end{array}$} & \multirow{3}{*}{$\begin{array}{c}\text { Cross } 1 \\
\text { (Giza40 } \\
\text { xGiza429) }\end{array}$} & $\mathrm{n}$ & 30 & 30 & 30 & 250 & 150 & 150 \\
\hline & & $\mathrm{X}$ & 6.16 & 6.23 & 6.04 & 5.92 & 5.86 & 5.94 \\
\hline & & $s^{2} \bar{X}$ & 0.010 & 0.009 & 0.007 & 0.009 & 0.011 & 0.010 \\
\hline & \multirow{3}{*}{$\begin{array}{l}\text { Cross } 2 \\
\text { (Sakha1 x } \\
\text { Giza429) } \\
\end{array}$} & $\mathrm{n}$ & 30 & 30 & 30 & 250 & 150 & 150 \\
\hline & & $\mathrm{X}$ & 3.01 & 6.23 & 4.89 & 5.75 & 4.38 & 5.85 \\
\hline & & $s^{2} \bar{X}$ & 0.006 & 0.009 & 0.006 & 0.005 & 0.005 & 0.006 \\
\hline & \multirow{3}{*}{$\begin{array}{c}\text { Cross } 3 \\
\text { (Giza } 429 \times \\
\text { TW) }\end{array}$} & $\mathrm{n}$ & 30 & 30 & 30 & 250 & 150 & 150 \\
\hline & & $\mathrm{X}$ & 6.23 & 6.15 & 5.98 & 5.91 & 6.01 & 6.00 \\
\hline & & $s^{2} \bar{X}$ & 0.009 & 0.016 & 0.024 & 0.006 & 0.006 & 0.007 \\
\hline \multirow{9}{*}{ Rust } & \multirow{3}{*}{$\begin{array}{c}\text { Cross } 1 \\
\text { (Giza40 } \\
\text { xGiza429) }\end{array}$} & $\mathrm{n}$ & 30 & 30 & 30 & 250 & 150 & 150 \\
\hline & & X & 6.61 & 6.56 & 6.14 & 6.18 & 6.54 & 6.48 \\
\hline & & $s^{2} X$ & 0.005 & 0.006 & 0.004 & 0.004 & 0.005 & 0.004 \\
\hline & \multirow{3}{*}{$\begin{array}{c}\text { Cross } 2 \\
\text { (Sakha1 x } \\
\text { Giza429) } \\
\end{array}$} & $\mathrm{n}$ & 30 & 30 & 30 & 250 & 150 & 150 \\
\hline & & $\mathrm{X}$ & 3.25 & 6.56 & 4.93 & 5.81 & 4.66 & 5.92 \\
\hline & & $s^{2} \overline{\mathbf{X}}$ & 0.005 & 0.006 & 0.006 & 0.004 & 0.005 & 0.004 \\
\hline & \multirow{3}{*}{$\begin{array}{c}\text { Cross } 3 \\
\text { (Giza } 429 \times \\
\text { TW) }\end{array}$} & $\mathrm{n}$ & 30 & 30 & 30 & 250 & 150 & 150 \\
\hline & & $\mathrm{X}$ & 6.56 & 6.97 & 6.04 & 6.00 & 6.14 & 6.28 \\
\hline & & $s^{2} \bar{X}$ & 0.006 & 0.014 & 0.021 & 0.005 & 0.005 & 0.006 \\
\hline
\end{tabular}


Table (4): Heterosis over mid (MP) and better (BP) parent and potence ratio (PR) for the studied traits in the three faba bean crosses.

\begin{tabular}{|c|c|c|c|c|c|c|c|c|c|c|}
\hline Characters & osses & MP & BP & PR & ID\% & $\mathrm{H} 2$ & h2(\%) & Ga\% & PCV\% & GCV\% \\
\hline \begin{tabular}{|l|l} 
Plant \\
\end{tabular} & 1 & 1.19 & -1.41 & 0.45 & 1.54 & 77.72 & 57.65 & 9.19 & 7.74 & 6.82 \\
\hline height & 2 & -0.85 & $-5.76^{\star \star}$ & -0.16 & 2.33 & 81.30 & 58.42 & 11.56 & 9.60 & 8.66 \\
\hline & 3 & $5.33^{* *}$ & $3.14^{* *}$ & 0.61 & -0.55 & 81.78 & 9.21 & 11.36 & 9.31 & 8.42 \\
\hline No. of & 1 & 2.39 & -3.92 & 0.36 & $25.36^{\star \star}$ & 73.23 & 52.71 & 60.43 & 55.66 & 47.63 \\
\hline brar & 2 & $22.78^{* *}$ & $\left|16.29^{* *}\right|$ & 4.08 & $16.95^{\star *}$ & 83.98 & 51.95 & $\mid 50.66$ & 47.34 & 43.38 \\
\hline plant & 3 & $47.73^{\star \star *}$ & $9.27^{\star *}$ & 1.36 & $4.39^{*}$ & 91.25 & 73.51 & 94.79 & 62.60 & 59.80 \\
\hline No. of & 1 & $19.05^{*}$ & $4.11^{*}$ & 1.33 & $25.76^{\star \star}$ & 84.73 & 59.53 & 24.86 & 20.27 & 18.66 \\
\hline pods/ plant & 2 & $17.75^{\star \star}$ & $14.41^{*}$ & 6.07 & $12.31^{\text {** }}$ & 92.84 & 83.66 & 76.41 & 44.347 & 42.72 \\
\hline & 3 & $41.71 / /$ & $14.99^{*}$ & 1.79 & $4.84^{*}$ & 95.52 & 71.04 & 63.53 & 43.41 & 42.43 \\
\hline No. & 1 & $4.42^{* \star}$ & $-4.30^{* *}$ & 0.49 & 11.42 & 77.86 & 57.36 & 8.53 & 7.22 & 6.37 \\
\hline & 2 & $15.51^{\star *}$ & $11.85^{\star *}$ & 4.74 & 9.17 & 92.12 & 39.43 & 10.12 & 12.46 & 11.96 \\
\hline plant & 3 & $56.12^{* *}$ & $6.87^{* *}$ & 1.22 & -3.99 & 84.00 & 62.18 & 11.14 & 8.70 & 7.97 \\
\hline Seed yield/ & 1 & $9.82^{\star \star}$ & 3.42 & 1.59 & 15.28 & 90.23 & 70.85 & 19.10 & 13.09 & 12.43 \\
\hline plant & 2 & $25.13^{\star \star}$ & $12.60^{* *}$ & 2.26 & 13.56 & 92.85 & 72.23 & 21.38 & 14.37 & 13.84 \\
\hline & 3 & $60.54^{\star *}$ & 1.76 & 1.08 & -4.89 & 87.31 & 60.96 & 16.66 & 13.27 & 12.40 \\
\hline No. & 1 & $-20.07^{\star \star}$ & $-24.92^{\star \star}$ & -3.33 & $-28.22^{* *}$ & 90.35 & 74.56 & 53.07 & 34.55 & 32.84 \\
\hline & 2 & -3.14 & $-4.05^{\star}$ & -2.00 & $-3.57^{\star *}$ & 84.77 & 79.31 & 47.77 & 29.24 & 27.09 \\
\hline & 3 & $20.32^{\star *}$ & $-4.98^{*}$ & 0.82 & $-4.26^{*}$ & 91.72 & 70.53 & 79.40 & 54.65 & 52.34 \\
\hline 100 seed & 1 & $4.90^{*}$ & 1.90 & -1.66 & 4.35 & 82.86 & 63.82 & 16.25 & 12.36 & 11.25 \\
\hline & 2 & $8.61^{* *}$ & 0.67 & 1.24 & 4.83 & 93.11 & 75.02 & 31.97 & 20.69 & 19.96 \\
\hline & 3 & $10.40^{\star \star}$ & $-4.77^{\star}$ & 0.74 & -0.86 & 76.00 & 66.02 & 20.79 & 15.28 & 13.32 \\
\hline Choc & 1 & -2.50 & -1.95 & -4.43 & 1.99 & 89.31 & 68.35 & 36.62 & 26.00 & 24.58 \\
\hline spot & 2 & $5.84^{\star *}$ & $62.46^{\star *}$ & 0.16 & $-17.59^{\star \star}$ & 82.49 & 45.76 & 17.81 & 18.89 & 17.16 \\
\hline & 3 & -3.39 & -2.76 & -5.25 & 1.17 & 85.75 & 68.97 & 28.95 & 20.37 & 16.61 \\
\hline Rust & 1 & $-6.76^{* *}$ & $-6.40^{\star *}$ & -17.80 & -0.65 & 85.71 & 66.67 & 22.77 & 16.58 & 15.35 \\
\hline & 2 & 0.51 & $51.69^{\star *}$ & 0.02 & $-17.85^{\star \star}$ & 83.33 & 74.04 & 26.77 & 17.55 & 16.02 \\
\hline & 3 & -10.72 & $-7.93^{\star \star}$ & -3.54 & & 83.59 & 77.34 & 30.04 & 18.86 & 15.55 \\
\hline
\end{tabular}

Heterosis:

Plant breeder have been investigated the possibility of developing hybrid cultivars. Thus the utilization of heterosis in various crops through the world has tremendously increased the production. Heterosis is a complex phenomenon which depends on the balance of different combinations of genotypes effect as well as the distribution of plus and minus alleles in parents. Heterosis is expressed as the percentage deviation of $F_{1}$ mean performance from the mid or better parent of the traits. As it will be expected, better parent from seed yield was the highest one. In this concern, percentage of heterosis over mid parent and better parent values is presented in the three crosses in Table (4) which indicated that heterosis over mid and better parent for plant height was significant in the third cross due to partial dominance, no. of branches and seeds/plants had highly significant mid and better parent heterosis in the second and third crosses due to over-dominance in most crosses, highly significant for no. of pods and seeds/plant in three crosses a result of over-dominance, highly significant for seed yield /plant in the first and second crosses due to over dominance, highly significant for no. of seeds/pod in the third cross due to partial dominance and highly significant in negative direction for rust disease in the first and third crosses due to over-dominance. However, the third cross (Giza 
$429 \times$ TW) showed significant better parental heterosis for plant height, no. of pods/plant, no. of seeds/plant, no. of seeds/pod and resistant to rust disease which give the cross the susceptibility over the other crosses. The differences in heterosis percent might be due to genetic variability of the parents and for non-allelic interactions, which can either increase or decrease the expression of heterosis. Even in the absence of epistasis, multiple alleles at a locus could lead to either positive of negative heterosis (Cress, 1966).

\section{Inbreeding depression:}

Inbreeding depression measured the extent of reduction of the $F_{2}$ generation due to inbreeding. Significant positive values were obtained for no. of branches/plant and no. of pods/plant in the three crosses. On the other hand, negative inbreeding depression values were obtained for no. of seeds/pod in the three crosses, reaction to chocolate spot and rust disease in the second cross (Sakha1 x Giza 429). The rest of traits over all crosses were non-significant. Significant effects for the both heterosis and inbreeding depression seem logic since the expression of heterosis in $F_{1}$ 's was followed by considerable reduction in the $F_{2}$ performance. Also, reduction in values of non-additive genetic components is expected caused by means of inbreeding depression. In addition, the conflicting estimates heterosis and inbreeding depression were associated in most traits. Similar conclusion were reviewed by El-Refaey and Radi (1991), El-Hady et al. (1998), Darwish et al. (2005), Attia and Salem (2006), Attia (2007), El-Hady et al. (2008), Abo Mostafa et al. (2009) and El- Hady et al. (2009).

\section{Heritability estimates:}

The highest broad sense heritability was obtained for no. of pods/plant in the third cross (Giza429 x TW) being (95.52\%) and 100-seed weight in the second cross (Sakha1 $x$ Giza429) where the value was $(93.11 \%)$ (Table 4). Meanwhile, the lowest estimates was resulted for no. of branches/plant in the first cross (Giza40 x Giza 429) with value of $73.23 \%$. Heritability in narrow sense as estimated by using $F_{2}$ and backcrosses data were low for no. of branches/plant, no. of seeds/plant and reaction to chocolate spot in the scond cross (Sakha1 x Giza429) with value of $51.95 \%$, $39.43 \%$ and $45.76 \%$, respectively. Meanwhile, high narrow sense values for number of pods/plant and no. of seeds/pod in the second cross (Sakha1 $\mathrm{x}$ Giza 429) with values $83.66 \%$ and $90.80 \%$, respectively. These results were in harmony with those obtained by Abdalla et al. (1999), Mansour et al. (2001), Darwish et al. (2005), El-Hady et al. (2007), El-Hady et al. (2009), Abou Mostafa et al. (2009) and Ashrei et al. (2013).

\section{Genetic advance:}

The genotypic coefficient of variability (GCV\%) and phenotyhpic coefficient (PCV\%), the predicted genetic advance upon selection as a percentage of $F_{2}$ generation for the studied characters are presented in Table (4). Number of branches/plant, no. of pods/plant and no. of seeds/pod in the three crosses exhibited high PCV\% and GCV\% and predicted genetic advance with high heritability. The highest genetic advance as mean percent $(\mathrm{Ga} \%)$ were detected for no. of branches/plant in the three crosses being $(60.43,50.66$ and $94.79 \%)$, respectively. Meanwhile, low predicted genetic advance values were obtained for plant height in the three crosses being 
(9.19, 11.56 and $11.36 \%)$, and for no. of seeds/plant in the three crosses $8.53,10.12$ and $11.14 \%$, respectively. Johanson et al. (1955) reported that, heritability estimates along with genetic advance are usually more useful than the heritability values alone in predicting the results of selecting the best individuals. In the present works high genetic advance was associated with high heritability values in narrow sense and $\mathrm{PCV} \%$ for no. of branches per plant, no. of pods/plant and no. of seeds/pod in the three crosses. Therefore, selection in these populations may be effective and satisfactory in the early generation (El-Refaey, 1999 and El-Hady et al. 2009). Also, moderate or low genetic advance was found to be associated with moderate or low heritability and PCV\% estimates. Therefore, it could be suggested that, the selection for faba bean seed yield in subsequent generation will be relatively more effective than in the early $F_{2}$ generation.

\section{Gene effects:}

The estimated values of different scaling test according to Mather (1949) and Hayman and Mather (1955), as well as six parameters describing the nature of gene action and their test of significance according to Gamble (1962), for all studied traits are presented in Table (5). (A) and (B) and tests provides evidence the presence of all types of non-allelic gene interaction. The significance of $C$ scale suggests (dd) type of epistasis. The significant $D$ scale reveals (aa) gene interaction, significance of $C$ and $D$ scales indicates (aa) and (dd) type of gene interaction. The test of adequacy of scales is important because in most cases the estimation of additive and dominance components of the variance are made assuming absence of gene interaction. The values of $A, B, C$ and $D$ should significantly differ than zero within the limits of their standard error. However, the results (Table 5) indicated that, the values of scaling test were significantly differ than zero for all studied traits in all crosses, except plant height and 100-seed weight of the first cross, no. of seeds/pod of the second cross and reaction to both chocolate spot and rust diseases of the third cross, and the reaction of chocolate spot in the first cross indicating that the additive dominance model is inadequate to interpret the gene effects (Mather, 1949), and simple additive- dominance model was adequate for estimating the genetic components of variance of these traits.

The estimated mean effect parameter $(m)$, which reflect the contribution due to the overall mean plus the locus effects and interaction of the fixed loci were highly significant in the three crosses of all traits. The additive gene effect (a) was significant in positive or negative direction in all crosses for all traits except in the third cross (Giza429 x TW) for no. of branches/plant, no. of pots and seeds/pod, and reaction to both chocolate spot and rust. In the second (Sakha1 x Giza 429) for no. of seeds/plant. For dominant effect (d) where its high in magnitude than that of additive type, because it gives high values comparing with additive gene (a) where were positive or negative directions. The values of dominant effect were significant in all crosses for all traits, except for no. of branches/plant, 100 seed weight and reaction to both chocolate and rust diseases in the third cross and for plant height and chocolate spot reaction in the first cross. These results indicated the importance role of dominance gene effects in the inheritance of these traits. On the other hand, significant of additive (a) and dominance (d) components indicated that, both additive and dominance gene 
effects are important in the inheritance of these traits. Also, selecting desirable characters may be practiced in the early generation but it would be effective in the late ones. Similar results were obtained by El-Hady et al. (2009), Abo Mostafa et al. (2009) and Ashrei et al. (2013) and El-Refaey and Abd El-Razek (2013). The additive $x$ additive gene effects was highly significant either positive or negative directions in all crosses for all studied traits except no. of seeds/pod in the first cross.

Table (5): Scaling test and gene action parameters of the studied traits in three faba bean crosses.

\begin{tabular}{|c|c|c|c|c|c|c|c|c|c|c|c|}
\hline \multirow[t]{2}{*}{ Characters } & \multirow{2}{*}{$\begin{array}{c}\text { Cross } \\
\text { es }\end{array}$} & \multicolumn{4}{|c|}{ Scaling test } & \multicolumn{6}{|c|}{ Gambles parameters } \\
\hline & & $\mathrm{A}$ & $\mathrm{B}$ & $\mathrm{C}$ & $\mathrm{D}$ & $(\mathrm{m})$ & (a) & (d) & (aa) & $(\mathrm{ad})$ & (dd) \\
\hline \multirow[t]{3}{*}{ Plant height } & 1 & 0.07 & -1.06 & -4.87 & -1.94 & 126.20 & 3.90 & 5.38 & 7 & - & \\
\hline & 2 & $12.77^{\star *}$ & 11.89 & $-14.22^{* *}$ & -19.44 & $126.00^{* *}$ & $7.22^{* *}$ & $37.77^{* *}$ & $38.88^{* *}$ & 0.44 & $-63.54^{* *}$ \\
\hline & 3 & $-18.09^{*}$ & $8.4^{\star}$ & $14.73^{* *}$ & 12.21 & $120.12^{* * t}$ & $-3.33^{*}$ & $-18.38^{* *}$ & $-24.42^{* *}$ & -13.25 & $34.11^{* *}$ \\
\hline \multirow{3}{*}{\begin{tabular}{|ll}
$\begin{array}{l}\text { No. } \\
\text { branches/ } \\
\text { plant }\end{array}$ & of \\
\end{tabular}} & 1 & -18 & -0.4 & -3.32 & -1.1 & 2.56 & 0.33 & 2.82 & 2.74 & 0.11 & -2.16 \\
\hline & 2 & $1.67^{\star *}$ & $0.9^{*}$ & $-1.25^{* *}$ & $-1.91^{* *}$ & $3.38^{* *}$ & $0.57^{\star}$ & $4.58^{* *}$ & $3.82^{* *}$ & $0.38^{\text {ns }}$ & -6.39 \\
\hline & 3 & $-1.11^{* *}$ & $1.04^{\star}$ & $1.61^{* *}$ & 0.84 & $3.27^{* *}$ & -0.26 & $-0.5^{\mathrm{s}}$ & $-1.68^{*}$ & $-1.08^{* *}$ & 1.75 \\
\hline \multirow{3}{*}{$\begin{array}{l}\text { No. of pods } \\
\text { plant }\end{array}$} & 1 & -4.25 & -11.16 & -37.23 & 9.09 & 38.90 & 9.77 & 30.21 & 21.8 & 3.46 & -6.41 \\
\hline & 2 & -5.96 & $9.37^{* *}$ & -8.73 & $-6.07^{* *}$ & $40.10^{\text {** }}$ & $-6.53^{*}$ & $19.04^{* *}$ & $12.14^{*}$ & $-7.67^{* \star}$ & -15.55 \\
\hline & 3 & $-12.33^{*+}$ & 0.57 & $17.12^{\text {t** }}$ & $14.44^{* *}$ & $41.25^{* *}$ & 0.66 & $-16.12^{*}$ & $-28.88^{* *}$ & $-6.45^{* *}$ & $40.64^{* *}$ \\
\hline \multirow{3}{*}{$\begin{array}{l}\text { No. of seeds/ } \\
\text { plant }\end{array}$} & 1 & 0.96 & -11.39 & $-50.43^{m}$ & -20.00 & 120.12 & $18.02^{\mathrm{m}}$ & $45.75^{\prime \prime}$ & 40.00 & $6.18^{\prime \prime}$ & -29.57 \\
\hline & 2 & $15.95^{* *}$ & $17.5^{* *}$ & $-13.87^{* *}$ & $-23.66^{* *}$ & $128.00^{* *}$ & 3.22 & $66.25^{*}$ & $47.32^{* *}$ & -0.77 & $-80.77^{* *}$ \\
\hline & 3 & $-93.77^{* *}$ & 2.76 & 110.79 & $100.9^{* *}$ & $131.15^{* *}$ & $-11.04^{* *}$ & -156.47 & -201.80 & -48.27 & 292.81 \\
\hline \multirow{3}{*}{$\begin{array}{ll}\text { Seed } & \text { yield } \\
\text { plant } & \end{array}$} & 1 & 4.15 & -0.94 & -40.91 & -17.66 & 80.20 & 12.28 & 43.79 & 35.32 & $6.95^{\prime \prime}$ & -29.73 \\
\hline & 2 & $32.46^{* *}$ & $11.74^{* *}$ & $-16.04^{* *}$ & -30.12 & $98.40^{* * *}$ & $20.48^{* *}$ & $83.10^{* *}$ & 60.24 & $10.36^{* *}$ & -104.44 \\
\hline & 3 & $-64.82^{* *}$ & 0.9 & & & $86.30^{* *}$ & $-3.25^{*}$ & -111.03 & -142.06 & -32.86 & 205.98 \\
\hline \multirow{3}{*}{$\begin{array}{l}\text { No. of seeds } \\
\text { pod }\end{array}$} & 1 & 0.43 & 0.52 & $1.51^{\prime \prime}$ & 0.28 & 3.09 & -0.24 & -1.17 & -0.56 & -0.0 & -0.39 \\
\hline & 2 & 0.87 & -0.33 & 0.24 & -0.15 & 3.1 & $0.57^{* *}$ & 0.20 & - & - & - \\
\hline & 3 & $-1.88^{* *}$ & 0.21 & $1.55^{* *}$ & $61^{*+}$ & $3.18^{* *}$ & -0.37 & $-2.71^{* *}$ & $-3.22^{* *}$ & $-1.05^{* *}$ & $4.89^{* *}$ \\
\hline \multirow{3}{*}{$\begin{array}{|ll|}100 & \text { seed } \\
\text { weight } & \\
\end{array}$} & 1 & 2.4 & -1.44 & -5.64 & -3.30 & $66.77^{\prime \prime}$ & -0.04 & 9.86 & - & - & - \\
\hline & 2 & $13.9^{* *}$ & -0.07 & -2.79 & $-7.77^{*}$ & $76.88^{* *}$ & $12.85^{* *}$ & $23.03^{*+}$ & $16.62^{* *}$ & $6.99^{* *}$ & $-30.45^{* *}$ \\
\hline & 3 & -2.97 & $6.66^{\star *}$ & $14.53^{* *}$ & $5.42^{*}$ & $65.80^{* *}$ & $4.60^{* *}$ & -4.69 & $-10.84^{\text {** }}$ & $-4.82^{* *}$ & 7.15 \\
\hline \multirow{3}{*}{$\begin{array}{l}\text { Chocolate } \\
\text { spot }\end{array}$} & 1 & -0.48 & -0.39 & -0.79 & 0.04 & 5.92 & -0.08 & -.23 & - & - & - \\
\hline & 2 & $0.86^{* *}$ & 0.58 & $3.98^{* *}$ & $1.27^{* *}$ & 5.75 & $-1.47^{* *}$ & $-2.27^{* *}$ & $-2.54^{* *}$ & 0.14 & 1.10 \\
\hline & 3 & -0.19 & -0.13 & -0.7 & -0.19 & 5.91 & 0.01 & 0.17 & - & - & - \\
\hline \multirow[t]{3}{*}{ Rust spot } & 1 & -.48 & -.39 & -0.79 & -0.66 & 6.18 & 0.06 & 0.88 & 1.32 & 0.03 & -1.91 \\
\hline & 2 & & & & & & $-1.26{ }^{* *}$ & $-2.06^{* *}$ & $-2.08^{* *}$ & $0.40^{* *}$ & $0 .-59$ \\
\hline & 3 & -0.32 & -0.45 & -1.61 & -0.42 & $6.00^{* *}$ & -0.14 & 0.11 & - & - & - \\
\hline
\end{tabular}

1= Giza40 x Giza 429, 2 = Sakha1 x Giza 429, 3= Giza429 x TW

However, significant positive epistatic gene action (ad) was observed in all crosses for all studied traits, except no. of branches/plant, no. of seeds/pod and reaction to rust disease in the first cross and plant height and no. of seeds/plant in the second cross where the values were not significant. The same trend was found with respect to dominance $x$ dominance (dd). While, positive or negative significant were observed for the remaining of the studied characters in these crosses. The absolute relative magnitude of the epistatic gene effects to the mean effects was somewhat variable depending on the cross and the studied traits. With regard to negative values observed either with main effects ; (a) and (d) or the non-allelic interactions i.e. (aa), (ad) and dd), this might indicate that, the alleles responsible for values traits was over dominant over the alleles controlling high value. Generally, the absolute magnitude of the epistatic effects was larger than additive or dominance effects in most cases. Therefore, it could 
be concluded that, homozygous $\mathrm{x}$ homozygous and heterozygous $\mathrm{x}$ homozygous non-allelic interactions were more important than the heterozygous $x$ heterozygous interaction in the inheritance of most studied traits. The epistatic gene effects were important than additive and dominance gene effects for most of the traits. The failure in detecting epistatic gene effects based on the generation mean analysis does no necessarily indicate that non-allelic interactions did not play role in the determination of phenotypic value. Nighawan et al. (1969) reported the importance of the three types of gene action in oats. Thus, the employed breeding system in exploiting any character depends on the involved gene action in its expression for predicted gain in selection progress (Abul-Naas et al., 1993). These results are in agreement with those reported by El-Hady et al. (1997), El-Refaey (1999), Kalia and Sood (2004), Attia et al. (2006), Al-Ghamdi (2007), El-Galaly et al. (2008) and El-Refaey and Abd ElRazek (2013).

\section{REFERENCES}

Abdalla, M.M.F.; M.M. El-Hady and E.H. El-Harty (1999). Investigations on faba bean (Vicia faba L.). 12 diallel crossed materials grown under cages.Proceed. First Plant Breed Conf., Egypt.J.Plant Breed., 3: 213229.

Abdelmula, A.A., Link, W., Kittlitz, E., von Stelling, D., (1999). Heterosis and inheritance of drought tolerance in faba bean (Vicia faba L). Plant Breed. 118, 485-490.

Abo Mostafa, R.A.I.; W.M. El-Rodeny and Aziza, M. Hassanein (2009). Generation mean analysis of earliness and chocolate spot disease resistance and quantitative variation in the storage proteins of three Vicia faba L. Crosses J. Agric. Sci., Mansoura Univ., 34(2): 925-943.

Abul-Naas, A.A.; M.A. Mahrous and A.A. El-Hosary (1993). Genetical studies on yield and some of its components in barley (Hordum vulgare L.). Egyhpt. J. Agron. 18(1-2): 33-46.

Al-Ghamdi, S.S. (2007). Genetic behavior of some selected faba bean genotypes. Proc. $8^{\text {th }}$ African Crop Science Society Conference, El-Minia, Egypt. pp. 709-714.

Ashrei, A.A.M.; Zeinab, E. Ghareeb; Kha, M.M. Ymani; Rehab, A.M. Abd Elrahman and A. Al-Garhy (2013). Statistical and genetical analysis of yield and some related components in faba bean. Egypt J. Plant Breed., 17(5): 57-67.

Attia, Sabah, M. (2007). Gene action and some genetic parameters for seed yield and its components in faba bean (Vicia faba L.). Egyptian J. of Appl. Sci., 22(6B): 487-499.

Attia, Sabah, M. and Manal Salem (2006). Analysis of yield and its components using diallel matings among five parents of faba bean. Egypt. J. Plant Breed., 10(1): 1-12.

Bernier, C.C., Hanounik, S.B., Hussein, M.M. and Mohamed, H.A. (1993). Field manual of common faba bean diseases in the Nile Valley. International Center for Agricultural Research in the Dry Areas (ICARDA) Information Bulletin No. 3. 
Bond, D.A., G.J. Jellis, G.G. Rowland, J. Le Guen, L.D. Robertson, S.A. Halil, and L. Li Juan. (1994). Present status and future strategy in breeding faba beans (Viciafaba L.) for resistance to biotic and abiotic stresses. Euphytica 73:151 -166.

Burton, G.W. (1952). Quantitative inheritance in grasses. Proceedings of the $6^{\text {th }}$ International Grassland Congress, August 17-23, 1952, Pennsylvania State College, USA, pp. 277-283.

Cress, C.E. (1966). Heterosis of the hybrid relative to gene frequency differences between two populations. Genetics, 53: 269-274.

Darwish, D.S.; M.M.F. Abdalla; M.M. El-Hady and S. El-Emam (2005). Investigation on faba beans, Vicia faba, L. 19-diallel and triallel matings using five parents. Proc. $4^{\text {th }}$ Plant Breed. Conf. March 5 (Suez Canal Univ.). Egypt. J. Plant Breed., 9(1): 197-208.

EL-Galaly, Ola_.A.M.; R.A.I. Abo Mostafa , and W.M. EL- Rodeny (2008), Evaluation of eight promising lines of faba bean (Vicia faba L.) for diseases resistance (chocolate spot and rust) and yield in North Delta . Annals of Agric . Sc., Moshtohor, Vol. 46(2): Ag. 131-140, (2008).

El-Hady, M.M.; A.M.A. Rizk; M.M. Omran and S.B. Ragheb (2007). Genetic behavior of some faba bean (Vicia faba, L.) genotypes and its crosses. Annals of Agric. Sci. Moshtohor, 45(1): 49-66.

El-Hady, M.M.; Gh.A. Gad El-Karim and M.A. Omar (1997). Genetical studies in faba bean (Viciafaba L.). J. Agric. Sci. Mansoura Univ. 22: 35613571.

El-Hady, M.M.; M.A. Omar; S.M. Naser; K.A. AH and M.S. Essa (1998). Gene action on seed yield and of some yield components in F] and F2 crosses among five faba bean (Viciafaba L.) genotypes. Bull. Fac. Agric., Cairo University, 49: 369-388.

El-Hady, M.M.; Sabah, M. Attia; A.A. Ashrei; E.A.A. El-Emam and M. Shaaban (2009). Heterosis, combining ability and genetic variability and some relative components in faba bean using six populations model. Minia J. Agric. Res. and Develop 29(3): 4187-431.

El-Hady, M.M.; Sabah, M. Attia; E.A.A. El-Emam; A.A.M. Ashrei and EM. Rabie (2008). Diallel mating among eight parents of faba bean (Vicia faba L.) and performance of $F_{1}$ and $F_{2}$.Egypt.J. of Appl.Sci.,23(5):95114.

El-Refaey, R.A. (1999). Generation mean analysis of earliness, yield and some of its components in three faba bean crosses. Minufiya J. Agric. Res. 24: 409-424.

El-Refaey, R.A. and M.M.Radi (1991) Genetic analysis of seed yield and its related character in soybean. II- Inbreeding depression and interrelationships among traits in the $\mathrm{Fl}$ and F2 generations. J.Agric. Res.Tanta Univ., 17(2):334-345.

El-Refaey, R.A. and U.A. Abd El-Razek (2013). Generation mean analysis for yield, its components and quality characteristics in four crosses of Egyptian cotton (Gossypium barbadense L.). Assion J. Crop Sci., 5(2): 153-166. 
Gamble, E.E., (1962).Gene effects in corn (Zea mays L.). I- Separation and relative importance of gene effects for yield.Can. J.of Plant Sci., 42: 339-348.

Hayman, B.I. and K. K. Mather (1955). The description of genetic interaction in continuous variation. Biometrics, 11: 69-82.

Hendawy,F.A (1994). Heterosis and inheritance of some quantitative characters in two intervarietal crosses of field bean (Viciafaba L.) Minufiya j.Agric .Res. 19:1957-1971

Jinks, J.L. and R.M. Jones (1958). Estimation of the components of heterosis. Genetics, 43: 223-234.

Johanson, H.W.; H.F. Robinson, and R.E. Comstock,(1955). Estimating of genetic and environmental variability in soybeans. Agron.J.,47: 314-318.

Kalia, P. and S. Sood (2004). Genetic variation and association analyses for pod yield and other agronomic and quality characters in an Indian himalayan collection of broad bean (Vicia Faba L.) . sabrao journal of breeding and genetics 36(2) 55-61.

Khalil, S.A.; M.M.El-Hady; R.F. Dissouky; M.I.Amer and S.A. Omer (1993). Breeding for high yielding ability with improved level of resistance to chocolate spot (Botrytis faba) diseases in faba bean (Vicia faba L.). J Agric. Sci . Mansoura Univ. 18(5) : 1315-1328.

Mansour, S.H.; Sabah, M. Attia; Manal M. Salem and M.M. El-Hady (2001). Genetic analysis of shedding and some yield characters in faba bean. J. Agric. Sci. Mansoura University, 26(4): 1875-1887.

Mansur, L.M.; A.L. Carriquiry and A.P. Rao-Arelli (1993). Generation mean analysis of resistance to race-3 of soybean cyst-nematode. Crop Sci., 33: 1249-1253.

Mather, K. (1949). Biometrical Genetics. Dover Publications, Inc., London Miller, P.A., J.C. Williams, H.F. Robinson and R.E. Comstock (1958). Estimates of genotypic and environmental variances and covariance in upland cotton and their implications in selection.Agron.J., 50: 126-131.

Miller, P.A.; J.C. Wiliams; H.F. Robinson and R.E. Comstock (1958). Estimates of genetic and envnironmental variance and covariance in upland cotton and their implication in selection. Agron. J., 50: 126-131.

Mohamed, H.A.; M.E. El-Rafei; N.M. Abou Zeid; S.A. Omer; Wadaia F. Habib.; LA. Ismail, ; M. Raof.; and H. Khidr; (1980). Plant pathobgy research studies ICARDA/IFAD Nile Valley Project on faba beans Annual coordination Meeting 1979-1980, Cairo, Mimeograph, 49pp.

Nighawan, Dechan and T.D. Yadava (1969). Detection of gene effect of some quantitative characters in oats (Avena sateral,L.).Indian J.Hered, 1: 145.

Singh, P. and S.S. Narayanan (1993). Biometrical Techniques in Plant Breeding. $1^{\text {st }}$ Edn. Kalyani Publishers, New Delhi, India, p. 182.

Stoddard, F.L; Nicholas, A; Rubiales, D; Thomas, J and Villegas-Ferna' ndez, A.M (2010). Integrated pest, disease and weed management in faba bean. Field Crops Res. 115, 308-318.

Warer, J.N. (1952). A method for estimating heritability. Agron. J. 44: 427430. 
تحليل متوسط الأجيال لصفات المقاومـة للأمر اض والمحصول ومكوناته فى ثلاثة

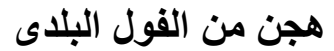

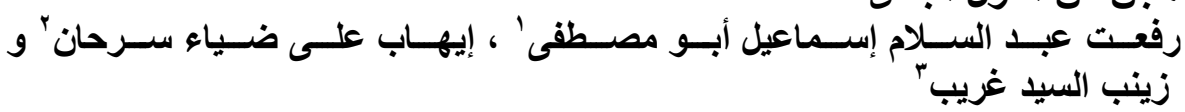

ا- برنـامج بحوث المحاصيل البقولية ـ معهد بحوث المحاصيل الحقلية ، مركز البحوث الزراعية ، جمهورية مصر العربية.

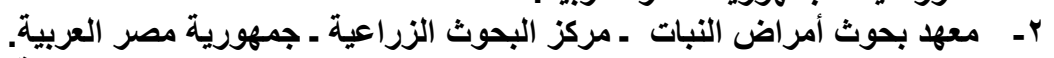

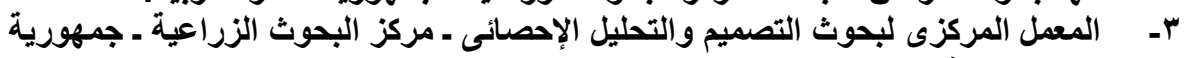
مصر العربية

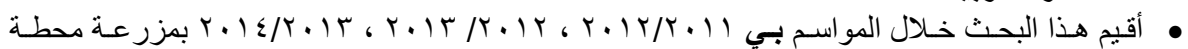
البحوث الزر اعية بسخا بمحافظة كفر الثيخ.

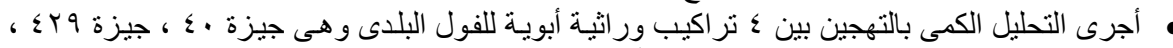

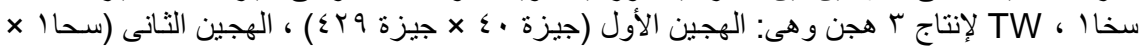

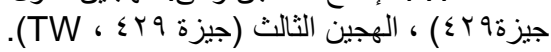

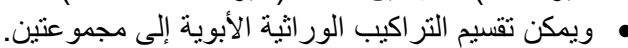

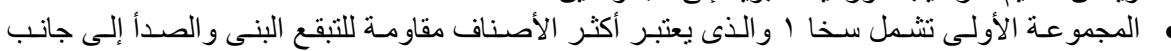

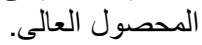

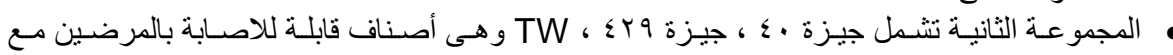
إنخفاض محصولهما.

• • أستخدم تحليل منوسط الأجيال لتقدير مكونات التباين الور اثي فى الهجن الثلاثة والتسعة صفات.

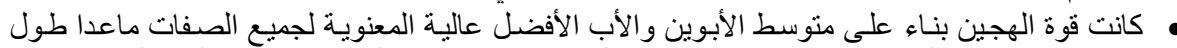

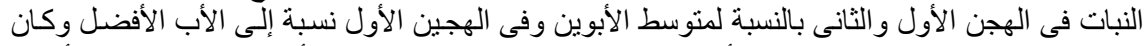

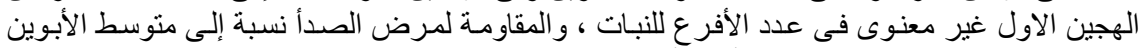

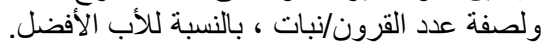

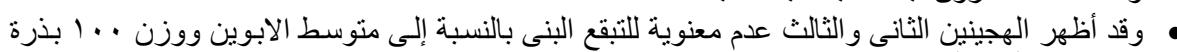

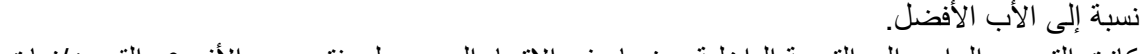
كانت التدهور الراجع إلى التربية الداخلية معنويا وفى الاتجاه الموجب لصفتى عدد الأفرع و القرون/نبات

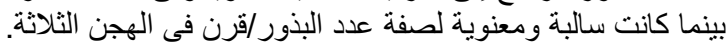

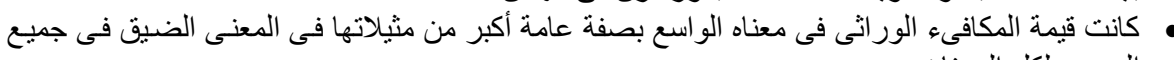

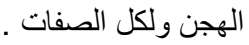
هانت الجينات المضيفة ذات أهية كبرى فى توريث معظم الصفات وبخاصة عدد القرون/نبات وعدد

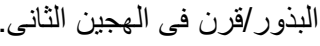

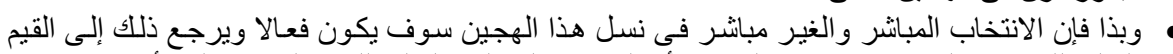

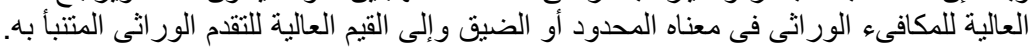

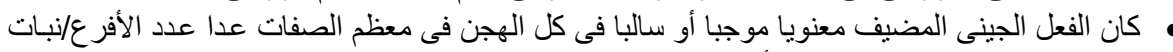

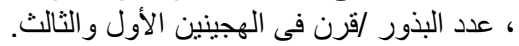

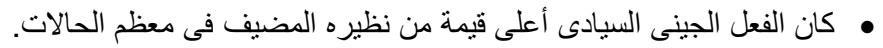
• كان الفعل الجينى الاضافى × الاضافى عالى المعنوية فى معظم الهجن وفى كل الصفير الصفات ماعدا حالات قليلة.

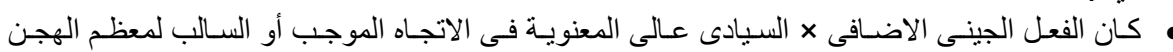
و ومغظم الصفات ونفس الاتجاه لوحظ بالنسبة للفعل الجينى السيادى × السيادى. 